\title{
Device for determining critical wind speed of stalk breaking to evaluate maize lodging resistance
}

\author{
Jun Xue, Bo Ming, Keru Wang, Ruizhi Xie, Peng Hou, Shaokun Li* \\ (Institute of Crop Sciences, Chinese Academy of Agricultural Sciences, Key Laboratory of Crop Physiology and Ecology, \\ Ministry of Agriculture and Rural Affairs, Beijing 100081, China)
}

\begin{abstract}
The accurate evaluation of the lodging resistance of maize plants can provide a basis for the breeding of lodging-resistant cultivars and the regulation of cultivation measures. However, the traditional methods for evaluating maize lodging resistance in terms of plant morphology and stalk mechanical strength have certain limitations. The objective of this research was to develop a device for determining the critical wind speed of maize stalk breaking. The device was equipped with a centrifugal fan to supply airflow and was powered by a frequency conversion motor. The frequency converter adjusted the motor speed and thus adjusted the wind speed. The wind speed decreased first and then increased with increasing height above the outlet of the device, and maximum wind speed can reach $40 \mathrm{~m} / \mathrm{s}$. This device was convenient for transportation in the field, has a low cost, and can quickly, accurately, and objectively determine the lodging resistance. Field tests showed that the device ran stably for a long time. The coefficient of variation of three repeated measurements was between $1.5 \%$ and $4.8 \%$ for four maize cultivars. The new device can measure the critical wind speed of maize lodging and identify the lodging resistance for different maize cultivars, cultivation practices, and plant health conditions, and can thus overcome barriers to measuring the maize lodging resistance under natural wind conditions.
\end{abstract}

Keywords: maize, stalk lodging, critical wind speed, turbofan, measuring device, wind pressure DOI: $10.25165 /$ j.ijabe.20201305.6033

Citation: Xue J, Ming B, Wang K R, Xie R Z, Hou P, Li S K. Device for determining critical wind speed of stalk breaking to evaluate maize lodging resistance. Int J Agric \& Biol Eng, 2020; 13(5): 1-7.

\section{Introduction}

Maize lodging reduces grain yield and quality and increases grain loss and harvest costs. Surveys have shown that lodging before maturity reduces annual maize yields by $5 \%-50 \%^{[1-3]}$. When lodging occurs after maturity, lodging increases the ear loss in mechanical grain harvesting. Previous study showed that ear loss in mechanical grain harvesting increased by $0.15 \%-0.59 \%$ for each $1 \%$ increase in the lodging percentage. The speed of mechanical grain harvesting has been found to decrease exponentially as the lodging percentage increases ${ }^{[4]}$. Therefore, improving the lodging resistance of maize is an important strategy for ensuring stable yields and facilitating mechanical grain harvesting.

Maize lodging is affected by many factors, including genotype, cultivation practices, and environmental conditions. Accurate evaluation of the lodging resistance of plants for different maize cultivars, cultivation practices, and environmental conditions can provide a basis for the breeding of lodging-resistant cultivars, the

Received date: $2020-07-23 \quad$ Accepted date: 2020-09-22

Biographies: Jun Xue, $\mathrm{PhD}$, research interest: maize lodging resistance, Email: xuejun5519@126.com; Bo Ming, PhD, Associate Professor, research interest: efficient utilization of environmental resources in crop production systems, Email: mingbo@caas.cn; Keru Wang, PhD, Professor, research interest: theory and technology of maize mechanization production, Email: wangkeru@caas.cn; Ruizhi Xie, PhD, Professor, research interest: maize physiology and ecology, Email: xieruizhi@caas.cn; Peng Hou, PhD, Associate Professor, research interest: physiology and ecology of maize high yield cultivation, Email: houpeng@caas.cn.

*Corresponding author: Shaokun Li, PhD, Professor, research interest: physiology and ecology of maize high yield and high efficiency cultivation. Institute of Crop Sciences, Chinese Academy of Agricultural Sciences, Beijing 100081, China. Tel: +86-10-82108891, Email: lishaokun@caas.cn. regulation of cultivation measures, and the selection of planting environment. Previous studies of maize lodging focused on aspects of plant height ${ }^{[5]}$, ear height, the height of the center of gravity $^{[6]}$, the length and diameter of the basal internodes ${ }^{[7]}$, stalk mechanical characteristics ${ }^{[8]}$, stalk anatomical structure, stalk carbohydrate accumulation and distribution ${ }^{[9]}$, vertical root-pulling resistance, root length density, average root diameter, and root depth and width ${ }^{[10,11]}$. Most of these studies were based on the resistance of the plant itself, while less consideration was given to the impact of the pushing force that is applied to the plant by the wind. Using a biomechanical model, Brune et al. ${ }^{[12]}$ showed that the primary factors affecting maize root lodging were root angle, structural rooting depth, soil strength, and wind speed; the secondary factors were plant height, ear height, leaf area, stalk taper, ear mass, and leaf drag; and the tertiary factors were stalk diameter and leaf number. Therefore, there are certain limitations to using the indicators of plant morphology and stalk mechanical strength to evaluate the lodging resistance of plants for different maize varieties and cultivation conditions, and a comprehensive indicator is thus needed to evaluate the lodging resistance of maize plants.

Wind is the primary environmental factor that is responsible for crop lodging. Maize lodging occurs when plants are subjected to wind forces that exceed the maximum force, the stalk and roots can withstand before breaking. $\mathrm{Li}$ et al. ${ }^{[13]}$ reported that strong wind during the pre- and post-flowering stages was responsible for $60 \%-99 \%$ of maize lodging. Some researchers have proposed to use environmental stress to test the lodging resistance of crop varieties $^{[14,15]}$. However, maize lodging often occurs under severe weather conditions, and lodging events are unpredictable in time and space. Therefore, it is often difficult to observe and record the field lodging process scientifically consequently, the critical 
wind speed of crop lodging is unclear. Any analysis of lodging is based on hypotheses of how lodging occurs that has not been fully substantiated $^{[12,14,16]}$. A wind tunnel is the most intuitive and practical means to determine the failure wind speed in order to evaluate crop lodging resistance. Portable wind tunnels were constructed in order to confirm its suitability for the quantitative investigation of the lodging process under various wind conditions and the evaluation of the accuracy of existing theoretical models of plant withdrawal ${ }^{[17,18]}$. The experimental results obtained using the tunnel were used to inform the breeding and cultivation of wheat and rice. However, these wind tunnels cannot be used to examine the effects of wind on maize lodging due to their limited wind speeds, which reach a maximum of $8.5 \mathrm{~m} / \mathrm{s}$ for the wheat ${ }^{[17]}$, and $16.6 \mathrm{~m} / \mathrm{s}$ for rice ${ }^{[18]}$. Maize is taller than wheat and rice, and thus the evaluation of maize lodging resistance requires a larger wind tunnel. However, large wind tunnels are inconvenient to move and most of them use indoor planting or potted plants. There are differences in light, temperature, and humidity between indoor environments and field environments, which result in differences in plant growth and development. Additionally, indoor planting has a relatively high cost and a relatively slow measurement speed. The DuPont Pioneer has devised a mobile wind machine that can generate wind speeds of up to $45 \mathrm{~m} / \mathrm{s}$ in order to assess stalk lodging under controlled wind conditions and thus facilitate the evaluation of a genotype for maize lodging ${ }^{[19]}$. Beijing Research Center for Information Technology in Agriculture designs and constructs a mobile wind machine to evaluate the lodging resistance of different cultivars and determine the relationship between lodging resistance and phenotypic traits. However, this mobile wind machine can only move in one direction and therefore the maize must be grown in a specific area ${ }^{[20]}$.

Therefore, this study sought to develop and evaluate a mobile wind machine, which was called the turbofan, to induce controlled maize lodging at the level of experimental field plots and the level of individual plants. Firstly, the natural stalk lodging in the field was analyzed. Secondly, according to the wind pressure and wind speed, which made the maize plant broken, the air outlet of the device was designed. Thirdly, the prototype of the device was fabricated. Finally, the stability and accuracy of the machine were tested by measuring the critical wind speed of stalk breaking (hereafter referred to as the "critical wind speed") for different maize cultivars, planting densities, and plant health conditions (presence or absence of stalk rot). This new device will be useful to inform the breeding of lodging-resistant maize cultivars and the formulation of cultivation practices.

\section{Analysis of natural lodging in the field}

In natural field conditions, maize lodging mostly occurs in the middle of the field (Figure 1). Computational fluid dynamics (CFD) simulation of airflow over a regular array of cubes showed that, the presence of a compensation flow through the side of the canopy, which enters the canopy from the upper part and exits from the lower part ${ }^{[21]}$. Crop canopy turbulence is often generated under natural conditions ${ }^{[22,23]}$, and plant bending stiffness plays an important role in the frequency and wavelength selection for the coherent motion of the canopy ${ }^{[24]}$. According to the above studies, a qualitative analysis of airflow in the field showed that, when the airflow I moves to position A, it is resisted by the maize plants, and thus the airflow I moves up to position B. When the airflow I reaches position $\mathrm{C}$, it collides with the parallel airflow II above the maize plants. Consequently, a downward force will occur at position $\mathrm{C}$, which causes the maize plants to bend. When the wind force reaches a critical value, the plants will break. Therefore, when maize lodging simulations are performed, the wind force received by the plant should be a top-down force.
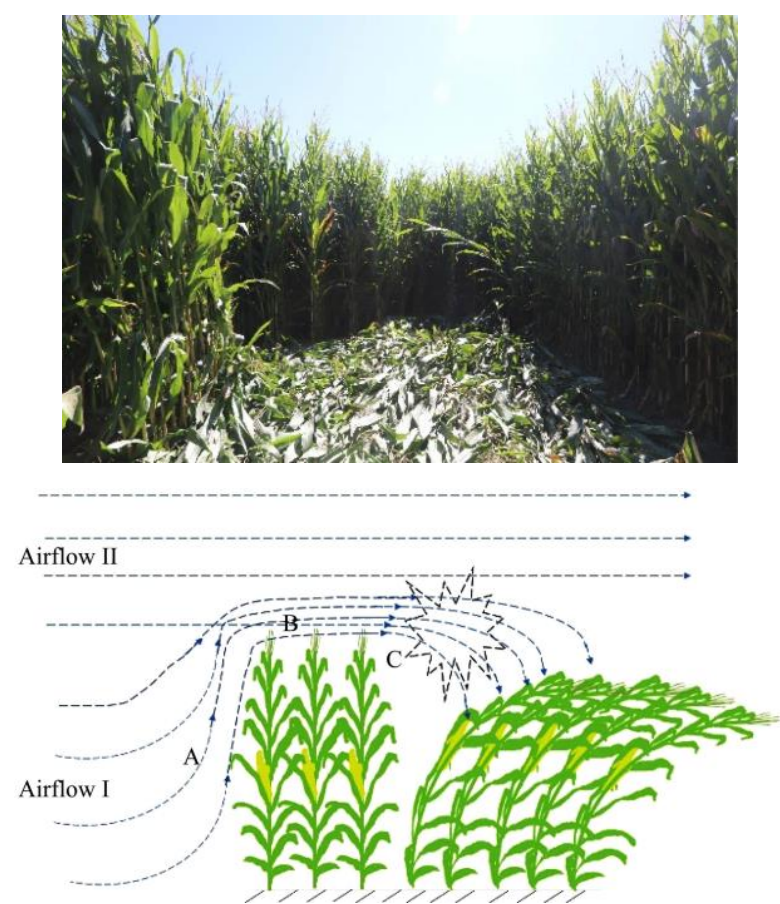

Figure 1 Analysis of lodging position and airflow in maize field

\section{Device description}

\subsection{Structure and composition}

The device consists of four subsystems: wind outlet equipment, a wind speed adjustment apparatus, a plant-fixing structure, and a wind speed measurement apparatus (an anemometer) (Figure 2). The wind outlet equipment is composed of a frequency conversion motor, a centrifugal fan, and a supporting structure. Four universal

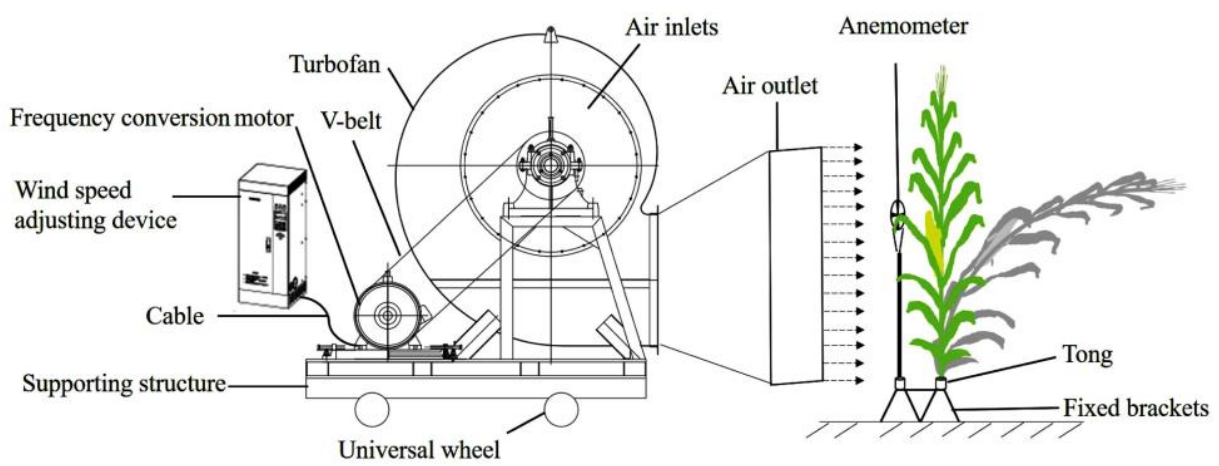

Figure 2 Structural diagram of the device for the measurement of maize lodging resistance 
wheels were installed under the supporting structure to facilitate movement in the field. The centrifugal fan is composed of a volute, a centrifugal blade, and a driveshaft. The centrifugal blade is connected to the driveshaft. The driveshaft is connected to the motor through four V-belts. The centrifugal fan has an air inlet on one side. The wind speed adjustment device is connected to the motor by a cable. The plant fixing structure is composed of a retractable bracket and a tong, which can be used to fix the basal internode of the maize stalk and the wind speed measuring apparatus. The heights of the plant and the anemometer can be adjusted using the retractable bracket.

\subsection{Wind pressure}

Maize plants bend when exposed to wind force, with the ear being the main destabilizing factor ${ }^{[25]}$. Previous study showed that the average breaking force $\left(F_{1}\right.$, Figure 3$)$ of 28 maize cultivars at the ear position was $23.0 \mathrm{~N}$ at the physiological maturity stage and that the average ear height $\left(h_{1}\right)$ of the same 28 maize cultivars was $84.3 \mathrm{~cm}^{[26]}$. According to Equation (1), the critical torque of stalk breaking was calculated to be $19.4 \mathrm{~N} \cdot \mathrm{m}$.

$$
T_{r}=F_{1} h_{1}
$$

The main maize organs which bear wind force are the leaves, the stalk, and the ear. Assume that the maize plants are evenly affected by wind force in the vertical direction. Previous study showed that the average plant height of the 28 maize cultivars was $246.7 \mathrm{~cm}^{[26]}$. According to Equation (2), the $F_{2}$ at half of the plant height $\left(h_{2}\right)$ was calculated to be $15.7 \mathrm{~N}$.

$$
F_{2}=T_{r} / h_{2}
$$

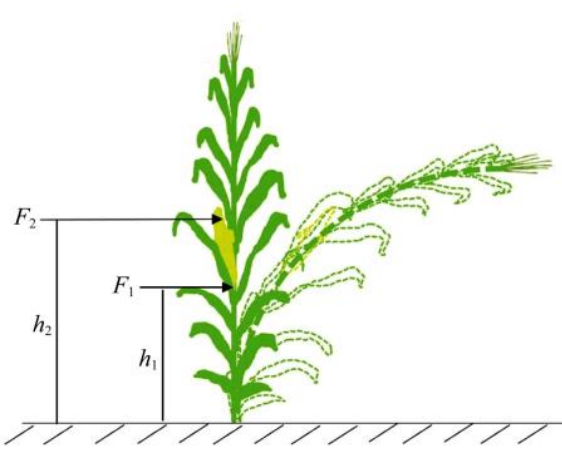

Figure 3 Schematic diagram of the effect of force on a maize plant

Under the normal planting density of $7.5 \times 10^{4}$ plants $/ \mathrm{hm}^{2}$, the average leaf area $\left(S_{1}\right)$ per plant was $5500 \mathrm{~cm}^{2}$, the average stalk diameter $\left(d_{1}\right)$ was $2.5 \mathrm{~cm}$, the average plant height $(h)$ was $246.7 \mathrm{~cm}$, and the average ear diameter $\left(d_{2}\right)$ was $6 \mathrm{~cm}$. The area of stalks and spikes calculated as cylinders, according to Equation (3), the total area $(S)$ per maize plant that is affected by the wind was $0.6675 \mathrm{~m}^{2}$.

$$
S=S_{1}+0.5 \pi d_{1} h+0.5 \pi d_{2} L
$$

According to Equation (4), the wind pressure $(P)$ at the outlet when the maize stalk is broken was $23.5 \mathrm{~N} / \mathrm{m}^{2}$. The maize leaves fall off after physiological maturity. When the leaf area is not considered, the wind pressure at the outlet was found to be $133.6 \mathrm{~N} / \mathrm{m}^{2}$. The plant was bent by wind force, when the half area of the stalk and ear was calculated using Equation (4), the wind pressure at the outlet was found to be $267.2 \mathrm{~N} / \mathrm{m}^{2}$. Therefore, the wind pressure $(P)$ at the outlet of the device needs to be higher than $267.2 \mathrm{~N} / \mathrm{m}^{2}$ to break the plants.

$$
P=F_{2} / S
$$

\subsection{Wind speed and airflow}

According to Bernoulli's Equation (Equation (5)), the wind speed caused stalks to break was $21.4 \mathrm{~m} / \mathrm{s}$.

$$
W_{p}=0.5 \times r_{o} \times V^{2}
$$

where, $W_{p}$ is the wind pressure, $267.2 \mathrm{~N} / \mathrm{m}^{2} ; V$ is the wind speed, $\mathrm{m} / \mathrm{s} ; r_{o}$ is the air density, $\mathrm{kg} / \mathrm{m}^{3}$. Under the $30^{\circ} \mathrm{C}$ and atmospheric pressure conditions, the air density is $1.165 \mathrm{~kg} / \mathrm{m}^{3}$.

At present, most of the maize production in China is planted with an equal row spacing of $60 \mathrm{~cm}$ or $80 \mathrm{~cm}+40 \mathrm{~cm}$ wide and narrow rows. Therefore, the width of the outlet set to $0.5 \mathrm{~m}$. Since the maize plant will be bent by the wind, the height of the outlet should be lower than the height of the plant; therefore, the height of the outlet set to $1.9 \mathrm{~m}$ (Figure 4). According to Equation (6), the airflow is calculated to be $1219.9 \mathrm{~m}^{3} / \mathrm{min}$.

$$
Q=60 \mathrm{~V} \times A
$$

where, $Q$ is the airflow, $\mathrm{m}^{3} / \mathrm{min}$ and $A$ is the cross-sectional area of the outlet, $\mathrm{m}^{2}$.

Therefore, at the outlet of the device, in order to break the plants, the wind speed needs to be higher than $21.4 \mathrm{~m} / \mathrm{s}$ and the airflow needs to be higher than $1219.9 \mathrm{~m}^{3} / \mathrm{min}$.

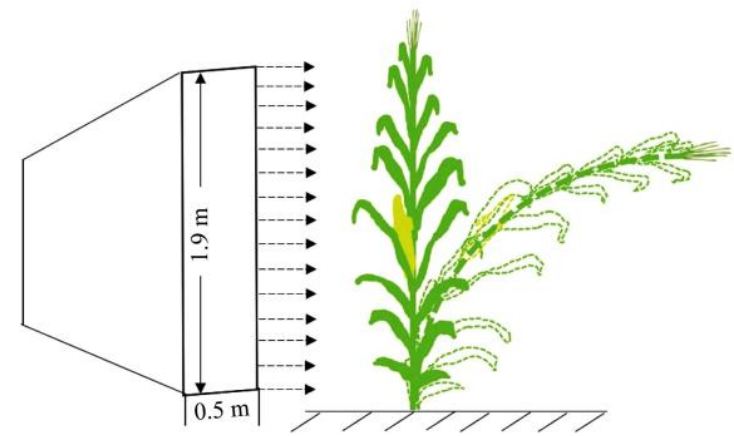

Figure 4 Schematic diagram of air outlet of the device

\subsection{Wind speed regulator}

The frequency converter was used to change the speed of the inverter motor and then change the speed of the turbofan, the wind pressure, and the wind speed. The frequency converter can be set to automatic or manual change. During automatic change, the time from $0 \mathrm{~Hz}$ to $50 \mathrm{~Hz}$ is $1 \mathrm{~min}$. Meanwhile, for manual change, step-less frequency conversion can be achieved by turning the knob. Frequency and wind speed obey the following linear relationship: $y=0.7052 x+0.0044, R^{2}=0.9996^{* *}$, where $y$ is wind speed, $x$ is frequency, and $* *$ indicates significance at $p<0.01$ level (Figure 5).

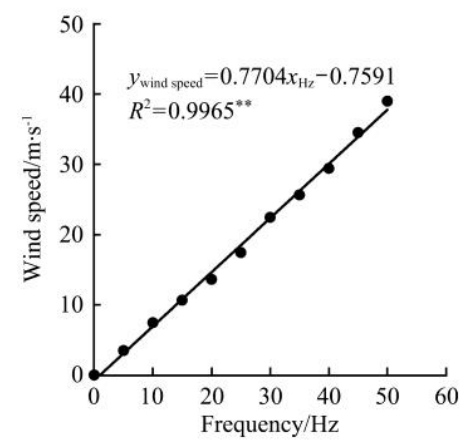

Figure 5 Relationship between electromotor frequency $(x)$ and wind speed $(y)$ at a horizontal distance of $30 \mathrm{~cm}$ and a vertical height of $120 \mathrm{~cm}$

\subsection{Performance of prototype}

According to the parameters of wind speed, wind pressure, and airflow, a centrifugal fan (G4-73 12C, Xinxiang Fan Company) with a turbine diameter of $1.4 \mathrm{~m}$ was combined with a frequency conversion motor. The input voltage of the inverter motor is $380 \mathrm{~V}$, the power is $55 \mathrm{~kW}$, and the maximum speed is $1100 \mathrm{r} / \mathrm{min}$. The total weight of the fan, motor, and supporting structure is about $2.8 \mathrm{t}$, which is convenient for transportation (Figure 6). 


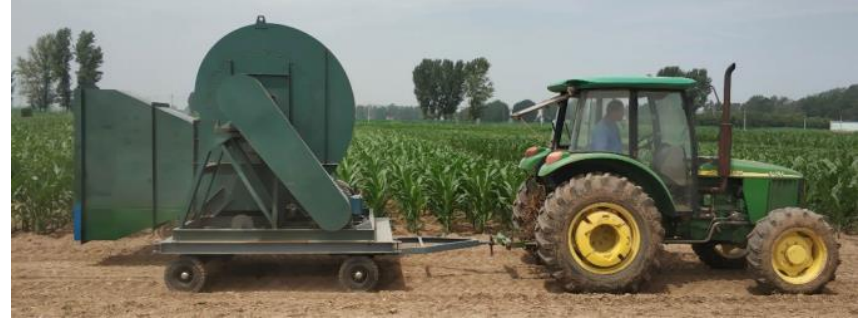

Figure 6 Prototype measuring device

The wind speeds from the fan outlets in the horizontal and vertical directions under different loads were measured. The 70 anemometers were evenly distributed, and the interval in horizontal and vertical directions was $30 \mathrm{~cm}$ (Figure 7a). The results showed that, in the horizontal direction, the wind speed decreased with increasing distance from the outlet, while in the vertical direction, the wind speed decreased first and then increased with increasing height above the outlet (Figures 7b-7f). This corresponds to the airflow pattern shown in Figure 1.

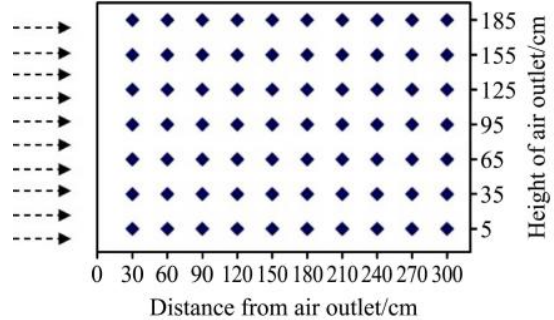

a. Anemometer

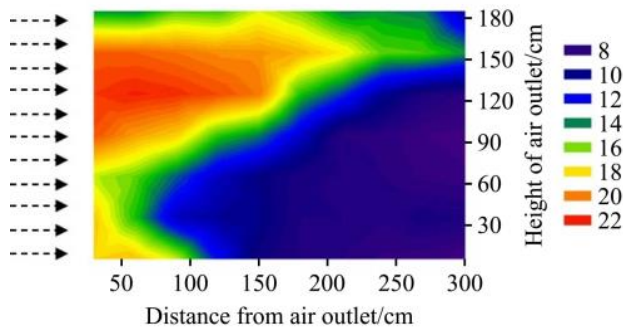

d. $60 \%$ load

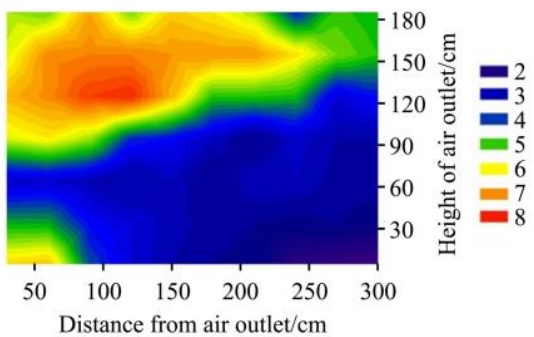

b. $20 \%$ load

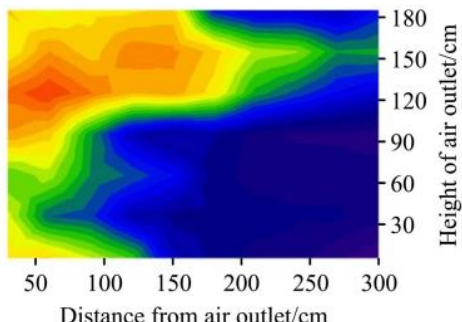

e. $80 \%$ load

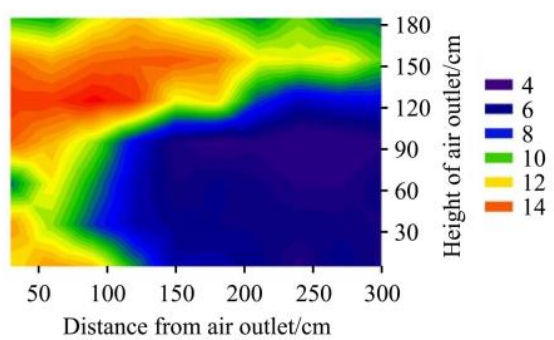

c. $40 \%$ load

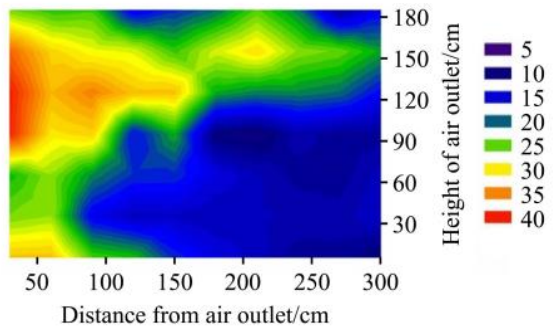

f. $100 \%$ load

Figure 7 Wind speed from the air outlet in the horizontal and vertical directions under different load conditions

\section{Field experiments}

\subsection{Experimental design}

Three field experiments were conducted at the Xinxiang Experimental Station of the Chinese Academy of Agricultural Sciences $\left(35^{\circ} 18^{\prime} \mathrm{N}, 113^{\circ} 54^{\prime} \mathrm{E}\right)$ during the 2018 maize growing seasons. Experiment 1: four widely planted maize cultivars, namely Zhengdan 958, Xianyu 335, Zhongdan 909, and Jingnongke 728, were planted during the 2018 summer-maize growing seasons. In China, the most widely planted maize cultivar is ZD958, followed by XY335, which accounted for $6.0 \%$ and $4.9 \%$ of the country's total maize planting area in 2016 , respectively ${ }^{[27]}$. Meanwhile, the planting area of the cultivar ZD909 was more than $66.7 \times 10^{4} \mathrm{hm}^{2}$ in $2016^{[28]}$. The cultivar JNK728 was developed to facilitate mechanical grain harvesting and high planting densities ${ }^{[29]}$. The plant density was $7.5 \times 10^{4}$ plants $/ \mathrm{hm}^{2}$. Experiment 2: five planting densities were used for Zhengdan 958 and Xianyu 335, which was 4.5, 6.0, 7.5, 9.0, and $10.5 \times 10^{4}$ plants $/ \mathrm{hm}^{2}$. A planting density of $7.5 \times 10^{4} \mathrm{plants} / \mathrm{hm}^{2}$ was used for Jingnongke 728 and Zhongdan 909. Experiment 3: the maize plants of health and stalk rot were randomly selected in natural field conditions. Stalk rot was determined in each maize cultivar by squeezing stalks between the first and second aboveground nodes ${ }^{[30]}$, and inspecting pith for discoloration of internodes as visible by darkening and pith deterioration ${ }^{[31]}$.

Each plot contained 10 rows with a length of $25 \mathrm{~m}$ and a row spacing of $60 \mathrm{~cm}$. All cultivars were arranged in randomized complete blocks. Each cultivar was replicated three times. The sowing date was June 13, 2018. The dates of silking and physiological maturity (PM) were recorded for each cultivar (Table 1). A controlled-release fertilizer was applied at
$156 \mathrm{~kg} \mathrm{~N} / \mathrm{hm}^{2}, 72 \mathrm{~kg} \mathrm{P}_{2} \mathrm{O}_{2} / \mathrm{hm}^{2}$, and $60 \mathrm{~kg} \mathrm{~K} 2 \mathrm{O} / \mathrm{hm}^{2}$ at sowing. Plants were irrigated according to the precipitation and water requirements of high-yield maize. Pesticides were applied as needed to control insect populations. Weeds were periodically removed by hand.

Table 1 Maize cultivar information in experiments

\begin{tabular}{llcc}
\hline \multicolumn{1}{c}{ Cultivar name } & \multicolumn{1}{c}{ Breeding department } & $\begin{array}{c}\text { Silking } \\
(\mathrm{m}-\mathrm{d})\end{array}$ & $\begin{array}{c}\text { PM } \\
(\mathrm{m}-\mathrm{d})\end{array}$ \\
\hline Zhengdan958 (ZD958) & $\begin{array}{l}\text { Henan Academy of Agricultural } \\
\text { Sciences }\end{array}$ & $8-5$ & $10-8$ \\
Xianyu335 (XY335) & $\begin{array}{l}\text { Pioneer Corporation, USA } \\
\text { Institute of Crop Sciences, Chinese } \\
\text { Zhongdan909 (ZD909) }\end{array}$ & $8-6$ & $10-7$ \\
$\begin{array}{l}\text { Academy of Agricultural Sciences } \\
\text { Jingnongke728 }\end{array}$ & $\begin{array}{l}\text { Beijing Academy of Agriculture } \\
\text { and Forestry Sciences }\end{array}$ & $8-3$ & $9-23$ \\
\hline
\end{tabular}

\subsection{Sampling and measurements}

Previous study showed that more than $90 \%$ of stalk lodging occurred between the second and fifth internodes above the soil ${ }^{[26]}$. Robertson et al. ${ }^{[32]}$ reported that more than $90 \%$ of stalk failure of maize was crease and the $2 \mathrm{~cm}$ above the node was the breaking point where most failure happens. In this study, three maize plants were randomly selected in each plot and cut at ground level. Each maize plant was fixed at the first internode of the stalk above the soil in order to ensure that the plant was oriented vertically. During the measurement, each plant was positioned $40 \mathrm{~cm}$ away from the air outlet with the bottom of the plant $30 \mathrm{~cm}$ above the bottom of the air outlet to ensure the ear within the range of maximum wind speed. The wind speed was then increased at a uniform rate until the stalk was broken. The critical wind speed of stalk breaking was read from the screen of the anemometer (Figure 8). 


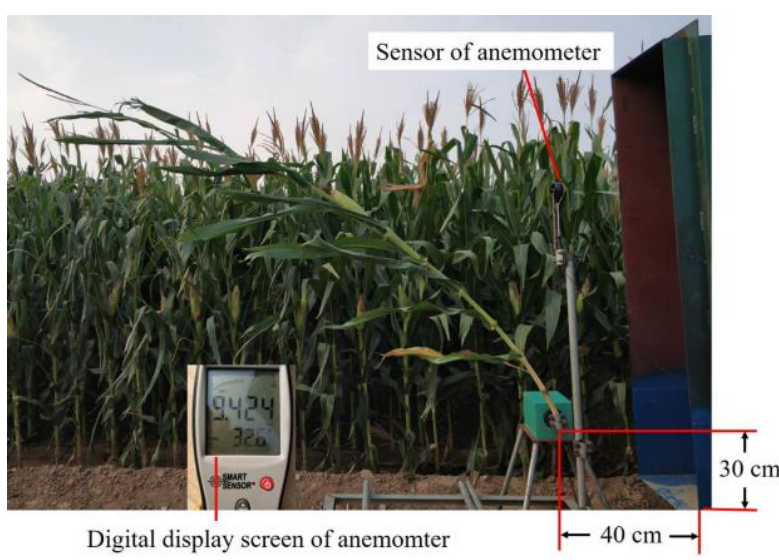

Figure 8 Prototype field test situation

\subsection{Statistical analyses}

Statistical analyses were performed using the Predictive Analytics Software (PASW) version 18.0 (IBM SPSS, Somers, NY). Data from each sampling date were analyzed separately. Means were tested using the least significant difference tests at the $p<0.05$ level (LSD 0.05).

\subsection{Experimental results}

4.4.1 Critical wind speed of stalk breaking in different maize cultivars

The critical wind speeds of the four cultivars are shown in Table 2. After physiological maturity, the critical wind speeds of cultivar Jingnongke 728 were significantly higher than those of the other three cultivars. The critical wind speeds of Zhengdan 958 were significantly lower than those of the other three cultivars. There was no significant difference in critical wind speed between Xianyu 335 and Zhongdan 909. This indicates that the new device can distinguish differences in the critical wind speed of stalk lodging among the different maize cultivars. The coefficient of variation among the three plant samplings ranged from $1.5 \%$ to $4.8 \%$ across the four maize cultivars, which indicates that the device can stably and reliably measure the critical wind speed of maize stalk lodging.

Table 2 Critical wind speeds of stalk lodging for different maize cultivars (October 12, 2018)

\begin{tabular}{lcccccc}
\hline \multirow{2}{*}{ Cultivar } & \multicolumn{3}{c}{ Critical wind speeds $/ \mathrm{m} \cdot \mathrm{s}^{-1}$} & \multicolumn{3}{c}{$\begin{array}{c}\text { Standard } \\
\text { deviation }\end{array}$} \\
\cline { 2 - 6 } & \multicolumn{3}{c}{ Sampling 1 } & Sampling 2 & Sampling 3 & Average \\
\hline Zhengdan 958 & 25.6 & 24.6 & 25.7 & $25.3 \mathrm{c}$ & 0.6 & 2.4 \\
Xianyu 335 & 32.1 & 29.8 & 32.7 & $31.5 \mathrm{~b}$ & 1.5 & 4.8 \\
Zhongdan 909 & 32.0 & 32.6 & 30.5 & $31.7 \mathrm{~b}$ & 1.1 & 3.4 \\
Jingnongke 728 & 37.3 & 36.9 & 38.0 & $37.4 \mathrm{a}$ & 0.6 & 1.5 \\
\hline
\end{tabular}

Note: Average values followed by different lowercase letters are significantly different among cultivars at the $p<0.05$ level. CV: coefficient of variation; RPS: rind penetration strength.

4.4.2 Effect of planting density on the critical wind speed of stalk breaking

The critical wind speed of maize stalk breaking significantly decreased as planting density increased (Figure 9). When the planting density was lower than $5.25 \times 10^{4}$ plants $/ \mathrm{hm}^{2}$, the critical wind speed of Zhengdan 958 was higher than that of Xianyu 335. When the planting density was higher than $5.25 \times 10^{4}$ plants $/ \mathrm{hm}^{2}$, the critical wind speed of Xianyu 335 was higher than that of Zhengdan 958. This indicates that the device can distinguish differences in the critical wind speed of stalk breaking among different cultivation conditions.

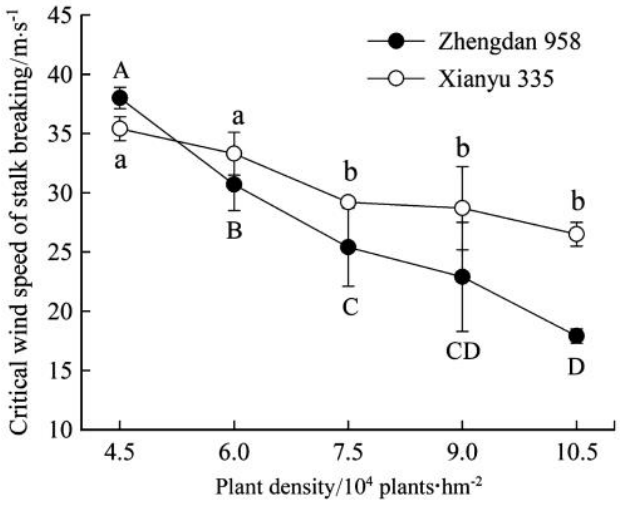

Note: Different uppercase letters indicate a significant difference at the $p<0.05$ level among different planting densities of Zhengdan 958 and different lowercase letters indicate a significant difference at the $p<0.05$ level among different planting densities of Xianyu 335.

Figure 9 Effect of planting density on the critical wind speed of maize stalk breaking (September 24, 2018)

4.4.3 Effect of stalk rot on the critical wind speed of stalk breaking

In natural field conditions, the critical wind speeds of maize stalk breaking of healthy plants were found to be significantly higher than those of plants with stalk rot (Figure 10).

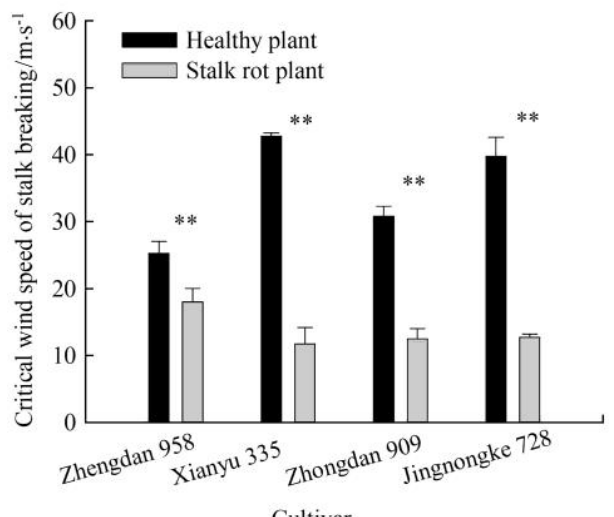

Cultivar

Note: $* *$ indicates significant difference at the $p<0.01$ level between healthy plants and stalk rot plants of the same cultivar.

Figure 10 Comparison of the critical wind speeds of stalk breaking between healthy plants and plants with stalk rot for different maize cultivars (October 27, 2018)

\section{Discussion}

Increasing the resistance of maize to lodging is an important goal of modern maize breeding. Accordingly, accurately judging the lodging resistance of maize cultivars is of great significance for breeders. Wind machines can be used to comprehensively evaluate the lodging resistance of maize plants, however current devices mostly use axial flow fans ${ }^{[17,18,20]}$. The results in winds spread sideways to the nearby open areas under wind machine tests. The difference in airflow for different outlet heights of the axial fan is small, which does not match the actual situation in the field, since, under natural conditions, the airflow pressure above the maize canopy is higher than that in the maize canopy ${ }^{[22]}$. This study first analyzed the law of natural maize lodging and the airflow pattern in field conditions. Additionally, a simple device was designed to measure the critical wind speed of stalk breaking. This device included a turbofan in order to make the airflow from the air outlet more similar to natural wind. In addition, this device has low cost, is convenient to move and simple to operate in the field, and has a high measurement efficiency and practicality. 
By measuring the critical wind speeds of stalk breaking for four maize cultivars, the stalk breaking occurred between the second and fourth internodes above the soil. The coefficient of variation between the three repeated measurements was lower than $5 \%$, it was shown that the prototype mobile wind machine is stable and has a controllable wind speed. Previous study showed that Zhengdan 958 and Zhongdan 909 have low stalk breaking force, but Xianyu 335 and Jingnongke 728 have high stalk breaking force ${ }^{[11]}$. This corresponds to the critical wind speed of four cultivars. As plant density increased, the stalk mechanical decreased, this caused the lodging rate to increase ${ }^{[33]}$. In this study, the critical wind speed of stalk breaking decreased as plant density. After physiological maturity, the stalk lodging rate increased as the stalk rot increased ${ }^{[31]}$. In this study, the critical wind speed of plants with stalk rot was found to be significantly lower than that of healthy plants. All of the above show that the turbofan can measure the critical wind speed of stalk breaking for different maize cultivars, cultivation practices, and plant health conditions. Maize lodging can occur at both the stalk and the root. Root lodging occurs most frequently during rainstorms. Daily precipitation is the main environmental determinant of maize lodging stress in the Huanghuaihai region ${ }^{[34]}$. The present research only involved a preliminary study on stalk breaking for different maize cultivars, planting densities, and plant health conditions, and the effect of wind and rain on maize lodging requires further study.

Wind is a random load whose speed and direction change over time. Before maize lodging, the plant is made to vibrate by the influence of wind ${ }^{[35]}$, and when the vibration of a maize plant exceeds a certain limit, the stalk breaks ${ }^{[36]}$. Maize lodging is affected by wind speed, wind direction, and the wind blowing time. This study only determined the critical wind speed of stalk breaking for individual maize plants in order to determine the stalk flexibility under different treatments. The wind direction, wind blowing time, and vibration frequency of the plant itself were not considered. Therefore, the obtained values of the critical wind speed of stalk breaking may be higher than the values in natural wind conditions. In order to study maize lodging, it is necessary to further study the heterogeneity of field airflow in time and space and develop a device that can simulate field airflow in the future.

\section{Conclusions}

A device was developed that can comprehensively evaluate the lodging resistance of maize in the field. The device is stable, easy to operate and move and can identify the lodging resistance of maize plants for different varieties and cultivation conditions. The device can overcome barriers to measuring the maize lodging resistance under natural wind conditions that the uncontrollability and randomness of wind speed and direction and the low efficiency of lodging identification.

\section{Acknowledgements}

This study was supported by the National Key Research and Development Program of China (Grant No. 2018YFD0100206), the Central Public-interest Scientific Institution Basal Research Fund (Grant No. Y2019PT07), the China Agriculture Research System (CARS-02-25), and the Agricultural Science and Technology Innovation Project of Chinese Academy of Agricultural Sciences.

\section{[References]}

[1] Zuber M S, Kang M S. Corn lodging slowed by sturdier stalks. Crops Soils, 1978; 30: 13-15.

[2] Minami M, Ujihara A. Effects of lodging on dry matter production, grain yield and nutritional composition at different growth stages in maize (Zea mays L.). Jpn J Crop Sci, 1991; 60: 107-115.

[3] Li S Y, Ma W, Peng J Y, Chen Z M. Study on yield loss of summer maize due to lodging at the big flare stage and grain filling stage. Sci Agric Sin, 2015; 19: 3952-3964. (in Chinese)

[4] Xue J, Li L L, Xie R Z, Wang K R, Hou P, Zhang W X, et al. Effect of lodging on maize grain losing and harvest efficiency in mechanical grain harvest. Acta Agron Sin, 2018; 44: 1774-1781. (in Chinese)

[5] Kamran M, Ahmad I, Wang H, Wu X, Jing X, Liu T, et al. Mepiquat chloride application increases lodging resistance of maize by enhancing stem physical strength and lignin biosynthesis. Field Crops Res, 2018; 224: $148-159$.

[6] Xu C, Gao Y, Tian B, Ren J, Meng Q, Wang P. Effects of EDAH, a novel plant growth regulator, on mechanical strength, stalk vascular bundles and grain yield of summer maize at high densities. Field Crops Res, 2017; 200: 71-79.

[7] Xue J, Xie R, Zhang W, Wang K, Hou P, Ming B, et al. Research progress on reduced lodging of high-yield and -density maize. Journal Integr Agr, 2017; 16: 2717-2725.

[8] Cook D D, Chapelle W, Lin T C, Lee S Y, Sun W, Robertson D J. DARLING: a device for assessing resistance to lodging in grain crops. Plant Methods, 2019; 15: 102. doi: 10.1186/s13007-019-0488-7.

[9] Xue J, Zhao Y, Gou L, Shi Z, Yao M, Zhang W. How high plant density of maize affects basal internode development and strength formation. Crop Sci, 2016; 56: 3295-3306.

[10] Bian D, Jia G, Cai L, Ma Z, Eneji A E, Cui Y. Effects of tillage practices on root characteristics and root lodging resistance of maize. Field Crops Res, 2015; 185: 89-96.

[11] Xue J, Gao S, Fan Y, Li L, Ming B, Wang K, et al. Traits of plant morphology, stalk mechanical strength, and biomass accumulation in the selection of lodging-resistant maize cultivars. Europ J Agron, 2020; 117: 126073. doi: 10.1016/j.eja.2020.126073.

[12] Brune P F, Baumgarten A, McKay S J, Technow F, Podhiny J J. A biomechanical model for maize root lodging. Plant Soil, 2018; 422: 397-408.

[13] Li S Y, Wang Y X, Hu C D, Yan Y. Effect of strong wind lodging at preand post-tasseling stages on growth and yield of summer maize. Chinese J Appl Ecol, 2015; 26: 2405-2413. (in Chinese)

[14] Berry P M, Sterling M, Mooney S J. Development of a model of lodging for barley. J Agron Crop Sci, 2006; 192: 151-158.

[15] Liu Z, Li S, Yang J, Yang Y, Mi C, Wang H, et al. Method of test environments selection for corn lodging resistance. Transaction of the CSAE, 2010; 26: 167-171. (in Chinese)

[16] Allcroft D J, Glasbey C A. Analysis of crop lodging using a latent variable model. J Agric Sci, 2003; 140: 383-393.

[17] Sterling M, Baker C J, Berry P M, Wade A. An experimental investigation of the lodging of wheat. Agricu Forest Meteorol, 2003; 119: 149-165.

[18] Shrestha S, Laza M R C, Mendez K V, Bhosale S, Dingkuhn M. The blaster: A methodology to induce rice lodging at plot scale to study lodging resistance. Field Crops Res, 2020; 245: 107663. doi: 10.1016/j.fcr.2019.107663.

[19] Steenhoek L, Carrigan L, Ghaffarzadeh M, Hartman M E, Barreiro R, Goldman DM, et al. Device and method for screening a plant population for wind damage resistance traits. 2008. U.S. Patent No. 7412880. Washington, DC: U.S. Patent and Trademark Office.

[20] Wen W, Gu S, Xiao B, Wang C, Wang J, Ma L, et al. In situ evaluation of stalk lodging resistance for different maize (Zea mays L.) cultivars using a mobile wind machine. Plant Methods, 2019; 15: 96. doi: 10.1186/s13007-019-0481-1.

[21] Santiago J L, Martilli A, Martín F. CFD simulation of airflow over a regular array of cubes. Part I: Three-dimensional simulation of the flow and validation with wind-tunnel measurements. Bound-Lay Meteorol, 2007; 122: 609-634.

[22] Finnigan J J. Turbulence in plant canopies. Annu Rev Fluid Mech, 2000; 32: 519-571.

[23] Py C, de Langre E, Moulia B, Hémon P. Measurement of wind-induced motion of crop canopies from digital video images. Agric Forest Meteorol, 2005; 130: 223-236. 
[24] Py C, de Langre E, Moulia B. A frequency lock-in mechanism in the interaction between wind and crop canopies. J Fluid Mech, 2006; 568: 425-449.

[25] Yuan Z H, Li Y D, Chen H S. Dynamic model and lodging resistance analysis of maize stem. J Maize Sci, 2002; 3: 74-75. (in Chinese)

[26] Xue J, Wang Q, Li L, Zhang W, Xie R, Wang K, et al. Changes of maize lodging after physiological maturity and its influencing factors. Acta Agron Sin, 2018; 44: 1782-1792 (in Chinese)

[27] Liu D F, Gao H C. Renewal of maize varieties is underway, which variety will replace 'Zhengdan 958' to become the largest variety? Southern Rural News, 2018-05-14. (in Chinese)

[28] National office for Science and Technology Awards. 2019 National science and technology progress award winning project directory. 2019. http://www.most.gov.cn/ztzl/gjkxjsjldh/jldh2019/jldh19jlgg/202001/t20200 103_150916.htm. Accessed on [2020-01-01].

[29] Duan M X, Zhao J R, Li Y F, Wang Y D, Xing J F, Zhang H S, et al. Study on the breeding and supporting technology of new maize variety 'Jingnongke 728'. J Agric, 2015; 5: 10-14. (in Chinese)
[30] Lipps P. Survival of colletotrichum graminicola in infested corn residues in Ohio, Plant Dis, 1983; 67: 102-104

[31] Thomison P R, Mullen R W, Lipps P E, Doerge T, Geyer A B. Corn response to harvest date as affected by plant population and hybrid. Agron J, 2011; 103: 1765-1772.

[32] Robertson D J, Julias M, Gardunia B W, Barten T, Cook D D. Corn stalk lodging: A forensic engineering approach provides insights into failure patterns and mechanisms. Crop Sci, 2015; 55: 2833-2841.

[33] Xue J, Gou L, Zhao Y, Yao M, Yao H, Tian J, et al. Effects of light intensity within the canopy on maize lodging. Field Crops Res, 2016; 188 133-141

[34] Yang Y, Yang J, Li S, Zhang X, Zhu D, Liu Z, et al. Spatial regression analysis on influence factors of maize lodging stress. Transaction of the CSAE, 2011; 27: 244-249. (in Chinese)

[35] Baker C J. The development of a theoretical model for the windthrow of plants. J Theor Biol, 1995; 175: 355-372.

[36] Flesch T K, Gran R H. Corn motion in the wind during senescence: I. motion characteristics. Agron J, 1992; 84: 742-747. 